\title{
Entropy of self-gravitating anisotropic matter
}

\author{
Hyeong-Chan Kim ${ }^{\mathrm{a}}$, Youngone Lee $^{\mathrm{b}}$ (I) \\ School of Liberal Arts and Sciences, Korea National University of Transportation, Chungju 380-702, Korea
}

Received: 11 March 2019 / Accepted: 1 August 2019 / Published online: 13 August 2019

(C) The Author(s) 2019

\begin{abstract}
We examine the entropy of self-gravitating anisotropic matter confined to a box in the context of general relativity. The configuration of self-gravitating matter is spherically symmetric, but has anisotropic pressure of which angular part is different from the radial part. We deduce the entropy from the relation between the thermodynamical laws and the continuity equation. The variational equation for this entropy is shown to reproduce the gravitational field equation for the anisotropic matter. This result re-assures us the correspondence between gravity and thermodynamics. We apply this method to calculate the entropies of a few objects such as compact star and wormholes.
\end{abstract}

\section{Introduction}

Ever since Hawking deduced the thermodynamics of black holes [1], thermodynamic approach to gravitating systems [2-6] provides good insights because it considers a relatively few thermodynamic variables rather than dealing complicated dynamic gravitational field equations. Getting thermodynamic quantities has thus been widely studied to understand gravitating systems. Examining self-gravitating systems has been one of those efforts for decades, which helps us to understand astrophysical systems. Especially, the entropy of a spherically symmetric self-gravitating radiation and its stability were calculated in a series of researches [7,8].

Those studies have shown that requiring maximum entropy of self-gravitating radiation in a spherical box reproduces the Tolman-Oppenheimer-Volkoff (TOV) equation for hydrostatic equilibrium $[9,10]$. This equivalence has been dubbed as 'Maximum Entropy Principle'(MEP). The total entropy is regarded as an action functional of mass, $m(r)$, the energy density $\rho(r)$ and the radius $r$. Varying the total entropy, one gets the hydrostatic equation. Hence, having exact entropy

\footnotetext{
a e-mail: hyeongchan@gmail.com

be-mail: youngone@ut.ac.kr
}

of a system enables one to get a hydrostatic equation through MEP. Recently, there have been discussions on the MEP in more general perspectives [11-13]. In most of those studies, the gravitating matters were assumed to be perfect fluids of which pressure $p(r)=w \rho(r)$ is locally isotropic. The entropy density for perfect fluids can be deduced from the continuity equation with series of thermodynamic equation combined [14]. It was given by a function of the energy density and $w$, that is, $s_{P} \equiv s_{P}(\rho, w)$.

When the energy distribution is smooth in a sufficiently small region of volume $V$, one can define energy density $\rho$ from $U(T, V)=\rho(T) V$ for the total energy $U$ inside the volume. Using the first law of thermodynamics, $T d S=$ $d U+p d V$, we have

$d S=\frac{V}{T} \frac{d \rho}{d T} d T+\frac{\rho+p}{T} d V$,

where $T$ and $S$ denote the temperature and the entropy of the system respectively. Since the entropy is a scalar quantity, the exactness condition of $S$ determines $\rho$ as a function of $T$ :

$\frac{\partial}{\partial T} \frac{\partial S}{\partial V}=\frac{\partial}{\partial V} \frac{\partial S}{\partial T} \longrightarrow \rho=\sigma(w) T^{\frac{1+w}{w}}$,

for a perfect fluid with $p=w \rho$, where $w$ is a constant. The entropy density of the fluid can be deduced by considering an isochoric process, for which $d U=T d S,{ }^{1}$

$$
\begin{aligned}
S & =(1+w) \sigma(w) T^{\frac{1}{w}} V+\text { constant } \\
& \rightarrow s_{P} \equiv \frac{S}{V}=\alpha_{w} \rho^{\frac{1}{1+w}}, \quad \alpha_{w}=(1+w) \sigma(w)^{\frac{w}{1+w}}
\end{aligned}
$$

where the integral constant can be set to zero by using the third law of thermodynamics. The constant $\alpha_{w}$ depends on the physical nature of matter consisting the fluid such as $w$.

\footnotetext{
1 The first law takes the form $T d S=d U+p d V$ if the volume of a system is allowed to change as in an expanding universe. Then, the entropy density is given by $s_{P}=2 \alpha_{w} \rho^{\frac{1}{1+w}}$. In either cases, the entropy density is proportional to $\rho^{\frac{1}{1+w}}$ up to a constant.
} 
This entropy density was already given in Refs. $[15,16]$. For radiation with $w=1 / 3$, the density $\rho_{\text {rad }}=\sigma_{\text {rad }} T^{4}$ and the constant $\sigma_{\text {rad }}$ is the Stefan-Boltzmann constant. ${ }^{2}$

Although majority of researches have focused on the dynamics of perfect fluid [17-19], anisotropic matter in cosmological configuration has recently drawn interests [2028]. For example, the authors in [29] obtained stable black holes with anisotropic matter. It is well known that, in general relativity, the throat of a traversable wormhole needs to be made of anisotropic matter [30,31]. Thus studies on the dynamics of anisotropic matter including thermodynamics become more important than ever.

For an anisotropic matter satisfying the linear equation of state,

$p_{k}=w_{k} \rho, \quad k=1,2,3$,

the entropy density $s_{A}$ will depend on $\rho$, and $w_{k}$ where the subscript $A$ denotes the 'anisotropic' nature. That is, $s_{A} \equiv$ $s_{A}\left(\rho, w_{1}, w_{2}, w_{3}\right)$. When all $w$ 's are equal, $s_{A}$ goes to that of the perfect fluid. Since an entropy density is a scalar quantity, $s_{A}$ can be written as a product of $s_{P}$ in Eq. (3) and a scalar function $\phi$ that describes the effect of anisotropy:

$$
\begin{aligned}
& s_{A}\left(\rho, w_{1}, w_{2}, w_{3}\right) \\
& \quad=\phi\left(w_{1}-w, w_{2}-w, w_{3}-w, \rho\right) \cdot s_{P}(\rho, w)
\end{aligned}
$$

where $\phi\left(w_{k}\right) \rightarrow 1$ in the isotropic limit. The function $\phi$ may also contain matter information which is not described by the equation of state.

In the above discussion, the pressure $\vec{p} \equiv\left(p_{1}, p_{2}, p_{3}\right)$ is divided into two parts. One is the isotropic part of pressure $\vec{p}_{\text {iso }} \equiv p_{\text {iso }}(1,1,1)$ and the other is the deviation from it, $\vec{p}_{\mathrm{d}} \equiv \vec{p}-\vec{p}_{\text {iso. }}$. However, there is an arbitrariness in the choice of the isotropic part. The first intuitive choice is $p_{\text {iso }} \equiv \bar{p}, \bar{p}=\left(p_{1}+p_{2}+p_{3}\right) / 3$. The other choice could be that $p_{\text {iso }} \equiv p_{1}$, and so on. The resulting entropy should be independent of this choice. This freedom will be gauged by a compensation vector later in this work.

We obtain the explicit form of the entropy density of selfgravitating anisotropic matter confined to a spherically symmetric box of radius $R$ from thermodynamic consideration up to a constant multiplication factor, which depends on the individual characteristics of matter. With this entropy density,

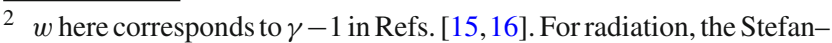
Boltzmann constant was derived by using the Planck's law, $\sigma_{\text {rad }}=$ $\frac{\pi^{2} k^{4}}{60 \hbar^{3} c^{2}}$. In Ref. [16], the entropy density was obtained for the special cases: (1) the thermal energy is much smaller than the Fermi energy in the core of neutron stars, (2) entropy density is proportional to number density, $s=\lambda n$, where $\lambda$ is a constant. The entropy density so obtained depends on the two constant $\gamma=w+1$ and a constant $K$. The author calculated $K$ for the case of radiation with $w=1 / 3$. In these cases, the constant $\sigma$ is a function of $\gamma$ and $K$, which can be calculated from the properties of radiation. It is not clear that the values of constant $\sigma$ are the same for all matters with the same $w$.
}

we perform the variation of total entropy of the anisotropic matter and show that the variational equation reproduces a modified TOV equation. The result of this article shows that MEP still holds. We also seek applications of this entropy to cosmological phenomena.

The order of the article is the following: We review properties of anisotropic matter in Sect. 2. In Sect. 3, the entropy of an anisotropic matter is obtained by comparing the continuity equation with thermodynamic relations. The MEP for a perfect fluid is briefly reviewed in Sect. 4 . The MEP yields the exact dynamical equation (modified TOV) using the entropy density of the anisotropic matter obtained in Sect. 3. We obtain explicitly the total entropy of a ball of anisotropic matter and apply this calculation to discuss properties of objects such as wormhole and a compact star in Sect. 5. Finally, we summarize our work and discuss about future issues in Sect. 6

\section{Anisotropic matter}

The stress-energy tensor $T^{a b}$ of a relativistic matter can be divided into a thermostatic part $T_{0}^{a b}$ and a dissipative part $T_{1}^{a b}$ as

$T^{a b}=T_{0}^{a b}+T_{1}^{a b}$.

The dissipative part is written as

$T_{1}^{a b}=u^{a} q^{b}+u^{b} q^{a}+\pi^{a b}, \quad u_{a} q^{a}=0$,

where $u^{a}$ is a timelike unit normal vector field. The quantities $q$ and $\pi^{a b}$ are interpreted as a heat flux vector and a viscous shear tensor, respectively. The viscous shear tensor satisfies $\pi^{a b} u_{b}=0$ or $u_{a} \pi^{a b} u_{b}=0$ for the Landau frame or the Eckart frame, respectively [14].

The thermostatic part of an isotropic perfect fluid has the form

$T_{0}^{a b}=\rho u^{a} u^{b}+p h^{a b}$,

$h^{a b}=x^{a} x^{b}+y^{a} y^{b}+z^{a} z^{b}=g^{a b}+u^{a} u^{b}$,

where $\rho$ and $p$ are the rest frame energy density and the isotropic pressure, respectively. The set of four mutually orthogonal vector fields $\left\{u^{a}, x^{a}, y^{a}, z^{a}\right\}$ conforms a frame of orthonormal vector fields. That is,

$g^{a b}=-u^{a} u^{b}+x^{a} x^{b}+y^{a} y^{b}+z^{a} z^{b}$,

$u_{a} u^{a}=-1, \quad x_{a} x^{a}=y_{a} y^{a}=z_{a} z^{a}=1$.

When matter is not perfect, more general tensor for anisotropic pressure replaces $T_{0}$, for example, elastic solid has anisotropic stress tensor [32]. In the absence of sheer, off-diagonal components of $\pi^{a b}$ vanishes. The energy- 
momentum tensor of the anisotropic matter being considered in this article has the form: ${ }^{3}$

$T_{A}^{a b} \equiv\left(T_{A}\right)_{0}^{a b}=\rho u^{a} u^{b}+p_{1} x^{a} x^{b}+p_{2} y^{a} y^{b}+p_{3} z^{a} z^{b}$,

where $\rho$ and $p_{k}(k=1,2,3)$ denote the energy density and the pressure measured in locally orthogonal rest frame, respectively.

A static, spherically symmetric metric can be written as $d s^{2}=-e^{u(r)} d t^{2}+e^{v(r)} d r^{2}+r^{2}\left(d \theta^{2}+\sin ^{2} \theta d \varphi^{2}\right)$.

Because of the spherical symmetry, we have $p_{\theta}=p_{\phi}$. We denote $p_{1} \equiv p_{r}$ and $p_{2} \equiv p_{\theta}=p_{\varphi}$ from now on. The Einstein equations $G_{t}^{t}=8 \pi T_{t}^{t}$ and $G_{r}^{r}=8 \pi T_{r}^{r}$ read

$e^{-v}=1-\frac{2 m(r)}{r}, \quad m(r) \equiv \int^{r} d r^{\prime} 4 \pi r^{2} \rho\left(r^{\prime}\right)$,

$u^{\prime}=\frac{2\left(m+4 \pi r^{3} p_{1}\right)}{r^{2}\left(1-\frac{2 m}{r}\right)}$.

With these equations and $G_{\theta}^{\theta}=8 \pi T_{\theta}^{\theta}$ gives the modified TOV equation:

$\frac{d p_{1}}{d r}=-\frac{\rho+p_{1}}{r(r-2 m)}\left(m+4 \pi r^{3} p_{1}\right)+\frac{2}{r}\left(p_{2}-p_{1}\right)$,

which resembles the TOV equation of the isotropic fluid [9, 10] up to the last term:

$\frac{d p}{d r}=-\frac{\rho+p}{r(r-2 m)}\left(m+4 \pi r^{3} p\right)$.

\section{Entropy density of anisotropic matter}

The temporal part of the continuity equation, $\nabla_{a} T_{A}^{a b}=0$, can be interpreted as a thermal relation

$$
-u_{b} \nabla_{a} T_{A}^{a b}=T \nabla_{a}\left(s_{A} u^{a}\right)+\mu_{N} \nabla_{a}\left(n u^{a}\right),
$$

here $T, s_{A}, n$ and $\mu_{N}$ denote the temperature, the entropy density, the number density and the chemical potential of the particles composing the anisotropic matter, respectively.

3 The anisotropic matter can also be regarded as an imperfect fluid. As we mentioned in Sect. 1, there is no preferred decomposition of the isotropic part in (11). For example, the isotropic part of the pressure can be $p$ or $p+\pi$ :

$$
\begin{aligned}
& {\left[\rho u^{a} u^{b}+p h^{a b}\right]+\pi^{a b}} \\
& \quad=\left[\rho u^{a} u^{b}+(p+\pi) h^{a b}\right]+\sigma^{a b}, \quad \pi \equiv \frac{1}{3} \operatorname{tr}\left(\pi^{a b}\right),
\end{aligned}
$$

where the terms in the bracket represent the isotropic part and $\sigma^{a b}$ is the traceless part of $\pi^{a b}$, i.e., $\pi^{a b}=\pi(x) h^{a b}+\sigma^{a b}$. One can find that $p$ and $\pi$ can have any values provided that they satisfy $p+\pi=$ $\left(p_{1}+p_{2}+p_{3}\right) / 3$.
In the presence of the dissipative part, $T_{1}^{a b} \neq 0$, the entropy vector $s_{A}^{a}$ can be defined $[14,33]$ :

$s_{A}^{a}=s_{A} u^{a}-\beta u_{b}\left(T_{1}^{a b}\right)$

where $\beta$ is the inverse temperature $\left(\beta \equiv T^{-1}\right)$. The net entropy production can be written as in Ref. [34],

$\nabla_{a} s_{A}^{a}=-\nabla_{(a} \beta u_{b)} \cdot T_{1}^{a b}$.

There are two ways for the entropy to be conserved. First, there exists a timelike Killing vector field $\xi^{a} \equiv \beta u^{a}$. Second, the dissipation is absent, $T_{1}=0$ or $T^{a b}=T_{A}^{a b}$. In this work, both of the two requirements hold.

When the number of particles does not change, $\nabla_{a}\left(n u^{a}\right)=$ 0 , the continuity equation reads:

$-u_{b} \nabla_{a} T_{A}^{a b}=T \nabla_{a}\left(s_{A} u^{a}\right)=0$.

Let us write the entropy density of anisotropic matter as $s_{A}=$ $\phi \cdot s_{P}$ as in Eq. (5). Here, $s_{P} \propto \rho^{\frac{1}{1+w}} u^{a}$ is the entropy density for a perfect fluid with an isotropic pressure $p_{\text {iso }}=w \rho$, and the function $\phi \equiv \phi\left(w_{1}-w, w_{2}-w, w_{3}-w\right)$ describes how much the entropy deviates from that of the isotropic form. Hence, $\phi=1$ when $w_{1}=w_{2}=w_{3}$, i.e., when isotropic.

The Eq. (19) becomes

$\nabla_{a} s_{A}^{a}=0 \rightarrow \nabla_{a}\left(s_{P}^{a}\right)=-\nabla_{a}(\log \phi) \cdot\left(s_{P}^{a}\right)$,

where $s_{A}^{a} \equiv s_{A} u^{a}$ and $s_{P}^{a} \equiv \alpha_{w} \rho^{\frac{1}{1+w}} u^{a}$. In general, the energy-momentum tensor (11) can be divided as:

$$
\begin{aligned}
T_{A}^{a b}= & T_{p}^{a b}+\left(p_{1}-p\right) x^{a} x^{b} \\
& +\left(p_{2}-p\right) y^{a} y^{b}+\left(p_{3}-p\right) z^{a} z^{b}, \\
T_{p}^{a b} \equiv & (\rho+p) u^{a} u^{b}+p g^{a b} .
\end{aligned}
$$

The continuity equation reads

$$
\begin{gathered}
-u_{b} \nabla_{a} T_{A}^{a b}=0 \rightarrow u \cdot \nabla \rho+(\rho+p) \nabla \cdot u \\
=\sum_{k=1}^{3}\left(p_{k}-p\right)\left(x_{k} \cdot \nabla x_{k}\right)_{a} u^{a} .
\end{gathered}
$$

The above equation can be written as a divergence relation of the entropy density vector of a perfect fluid $s_{P} \propto \rho^{1 /(1+w)} u^{a}$ :

$\nabla_{a}\left(\rho^{\frac{1}{1+w}} u^{a}\right)=\sum_{k=1}^{3} \frac{w_{k}-w}{1+w}\left(x_{k} \cdot \nabla x_{k}\right)_{a}\left(\rho^{\frac{1}{1+w_{1}}} u^{a}\right)$.

The case when $w=-1(\rho+p=0)$ will be treated separately. Comparing this equation with Eq. (20) gives

$$
\begin{aligned}
\nabla_{a}(\log \phi) \\
=\left[\frac{w_{1}-w}{1+w} x \cdot \nabla x_{a}+\frac{w_{2}-w}{1+w} y \cdot \nabla y_{a}+\frac{w_{3}-w}{1+w} z \cdot \nabla z_{a}\right] \\
\quad+\Phi_{a}(x)
\end{aligned}
$$


where $\Phi_{a}(x)$ is the compensation vector mentioned in Sect. 1. It is a vector orthogonal to $u^{a}\left(\Phi_{a} u^{a}=0\right)$ and represents the anisotropic effect. In other words, $\Phi_{a}=0$ when matter is isotropic.

For a spherically symmetric configuration, we can take $x^{a}, y^{a}, z^{a}$ as unit radial, axial and azimuthal spacelike vectors, respectively. In this case, for the metric (12), a calculation in the orthonormal basis

$u^{a}=e^{-u / 2}\left(\frac{\partial}{\partial t}\right)^{a}, \quad x^{a}=e^{-v / 2}\left(\frac{\partial}{\partial r}\right)^{a}$,

$y^{a}=\frac{1}{r}\left(\frac{\partial}{\partial \theta}\right)^{a}, \quad z^{a}=\frac{1}{r \sin \theta}\left(\frac{\partial}{\partial \varphi}\right)^{a}$,

gives

$$
\begin{array}{r}
x \cdot \nabla x_{a}=0, \quad y \cdot \nabla y_{a}=-\frac{1}{r} \delta_{a r}, \\
z \cdot \nabla z_{a}=-\frac{1}{r} \delta_{a r}-\cot \theta \delta_{a \theta} .
\end{array}
$$

With $w_{2}=w_{\theta}=w_{\phi}$, one gets from (24) and (26),

$\partial_{t}(\log \phi)=\Phi_{t}$

$\partial_{r}(\log \phi)=\frac{2}{r} \frac{w_{2}-w}{1+w}+\Phi_{r}$,

$\partial_{\theta}(\log \phi)=\cot \theta+\Phi_{\theta}$,

$\partial_{\varphi}(\log \phi)=\Phi_{\varphi}$.

Since the factor $\phi$ depends only on the radial coordinate, one can determine the components of $\Phi_{a}$ to be $\Phi_{t}=0, \Phi_{\theta}=$ $-\cot \theta, \Phi_{\varphi}=0$ and $\Phi_{r}=\Phi_{r}(r)$ without loss of generality. Therefore, one function $\Phi_{r}$ is sufficient to gauge the difference between two isotropic pressures. Solving the equations gives

$\phi=C\left(w_{2}-w\right) \Phi_{w}(r) r^{\frac{2\left(w_{2}-w\right)}{1+w}}$,

where $C$ is an integral constant and $\Phi_{w}(r) \equiv e^{\int \Phi_{r}}$ is a function to be determined. We call this function $\Phi_{w}$ a compensation factor. Therefore, in general, the anisotropic entropy density has the form:

$s_{A}=\alpha_{w} \Phi_{w}(r) \rho^{\frac{1}{1+w}} r^{\frac{2\left(w_{2}-w\right)}{1+w}}$.

The entropy density is a function of energy density and other related parameters ( $w$ 's here). Because entropy density is an intensive quantity, the $r$ dependence above seems awkward at first sight. Actually, the entropy density can be written as a function of energy density, $s_{A} \equiv s_{A}(\rho)$. One can regard $r$ as a function of $\rho$ since $\rho$ satisfies a first order differential equation

$\frac{d \rho}{d r}=f(u(r), v(r), \rho, r)$

for a given metric $u, v$ in Eq. (12). Given a specific $u(r)$ and $v(r)$, the above equation solves $r$ as a function of energy density, $r=r(\rho)$.
If one knows a compensation factor $\Phi_{w}(r)$ for a choice of isotropic pressure, $p_{\text {iso }}=w \rho$, the compensation factor $\Phi_{w^{\prime}}(r)$ for other choice $p_{\text {iso }}^{\prime}=w^{\prime} \rho$ can be calculated. By equating entropies $s_{A}\left(w, w_{2}\right)=s_{A}\left(w^{\prime}, w_{2}\right)$,

$\alpha_{w} \Phi_{w}(r) \rho^{\frac{1}{1+w}} r^{\frac{2\left(w_{2}-w\right)}{1+w}}=\alpha_{w^{\prime}} \Phi_{w^{\prime}}(r) \rho^{\frac{1}{1+w^{\prime}}} r^{\frac{2\left(w_{2}-w^{\prime}\right)}{1+w^{\prime}}}$,

one gets $\Phi_{w^{\prime}}$ in terms of $\Phi_{w}$. Obtaining a compensation factor $\Phi_{w}$ for a specific choice which gives a modified TOV in Eq. (14) is not always an easy task. However, if there is a choice $w_{0}$ which makes $\Phi_{w_{0}}$ be a constant, a general compensation factor will have the form:

$\Phi_{w}(r) \propto\left[\rho(r) r^{2\left(1+w_{2}\right)}\right]^{\frac{w-w_{0}}{(1+w)\left(1+w_{0}\right)}}$.

We will show that, with a choice $\vec{p}_{\text {iso }}=\vec{p}_{1}$, i.e. $w_{0}=w_{1}$, the MEP with a constant $\Phi$ produces the exact modified TOV equation. Consequently, one can write down the entropy density of anisotropic matter as:

$s_{A}=\alpha_{\left(w_{1}, w_{2}\right)} \rho^{\frac{1}{1+w_{1}}} r^{\frac{2\left(w_{2}-w_{1}\right)}{1+w_{1}}}$,

where $\alpha_{\left(w_{1}, w_{2}\right)}$ is a constant that does not modify the equation of motion and is determined by the physical nature of anisotropic matter as we mentioned above in Ref. [16].

When $\rho+p_{1}=0$ or $w_{1}=-1$, it is obvious that one cannot use the formula (33) directly. Thankfully, one can use the freedom of choice for the isotropic part to solve this problem. As an example, we obtain an explicit form of the compensation factor for $w_{1}=-1$ in the next section.

\section{Requiring maximum entropy}

The local maximum of entropy of relativistic matter coincides with a dynamically stable equilibrium configuration (MEP) [7,8]. The premises in those papers about extrema of total entropy also work here. The system being considered in this article is static and spherically symmetric. The only difference is that the pressure of matter is anisotropic. Thus, all the arguments used in Ref. [8] about extrinsic curvature $K_{a b}$ of a spacelike hyper-surface $\Sigma$ holds here.

In this section, we briefly review the MEP for an isotropic matter (perfect fluid) and then generalize to the anisotropic matter case. Finally, for the exceptional case with $w_{1}=-1$, we obtain the entropy density separately.

\subsection{Isotropic matter case}

The total entropy of the isotropic fluid in a spherical box of radius $R$ is given by 


$$
\begin{aligned}
S \equiv \int d \Sigma s_{P}^{a} n_{a}= & 4 \pi \alpha_{w} \int_{0}^{R} d r \frac{r^{2}}{\sqrt{1-\frac{2 m(r)}{r}}} \rho^{\frac{1}{1+w}} \\
= & (4 \pi)^{\frac{w}{1+w}} \alpha_{w} \int_{0}^{R} d r m^{\frac{1}{1+w_{1}}} r^{2\left(1-\frac{1}{1+w_{1}}\right)} \\
& \times\left(1-\frac{2 m}{r}\right)^{-\frac{1}{2}}
\end{aligned}
$$

The total entropy can be regarded as an action integral $I=\int_{0}^{R} d r \mathcal{L}_{0}\left[m(r), m^{\prime}(r) ; r\right]$ where the corresponding Lagrangian is

$$
\begin{aligned}
\mathcal{L}_{0}\left[m(r), m^{\prime}(r) ; r\right] \equiv & (4 \pi)^{\frac{w}{1+w}} \alpha_{w} m^{\frac{1}{1+w_{1}}} r^{2\left(1-\frac{1}{1+w_{1}}\right)} \\
& \times\left(1-\frac{2 m}{r}\right)^{-\frac{1}{2}} .
\end{aligned}
$$

As expected, the Euler-Lagrange equation, $\frac{d}{d r}\left(\frac{\partial \mathcal{L}_{0}}{\partial m^{\prime}}\right)-$ $\frac{\partial \mathcal{L}_{0}}{\partial m}=0$, gives the original TOV equation (15).

\subsection{Anisotropic matter when $w_{1} \neq-1$}

For anisotropic matter with static, spherically symmetric distribution, in accordance with MEP, the variation of total entropy gives the exact modified TOV equation (14). The total entropy of the anisotropic matter in a box of radius $R$ is given by

$$
\begin{aligned}
S_{A} & =\int d \Sigma s_{A}^{a} n_{a} \\
& =4 \pi \alpha_{w} \int_{0}^{R} d r \frac{r^{2}}{\sqrt{1-\frac{2 m(r)}{r}}} r^{\frac{2\left(w_{2}-w_{1}\right)}{1+w_{1}}} \rho^{\frac{1}{1+w_{1}}},
\end{aligned}
$$

where we use the entropy density in Eq. (33) with $\Phi_{w}=1$. In this case, the action to be extremized is given by

$$
\begin{aligned}
I & =\int_{0}^{R} d r \mathcal{L}\left(m, m^{\prime} ; r\right), \\
\mathcal{L}\left(m, m^{\prime} ; r\right) & \equiv r^{\frac{2\left(w_{2}-w_{1}\right)}{1+w_{1}}} \mathcal{L}_{0}\left(m, m^{\prime} ; r\right) \\
& =(4 \pi)^{\frac{w}{1+w}} \alpha_{w}\left(m^{\prime}\right)^{\frac{1}{1+w_{1}}} r^{\frac{2 w_{2}}{1+w_{1}}}\left(1-\frac{2 m}{r}\right)^{-\frac{1}{2}},
\end{aligned}
$$

where $\mathcal{L}_{0}$ is the Lagrangian for the isotropic part in Eq. (35). The Euler-Lagrange equation

$$
\frac{d}{d r}\left(\frac{\partial \mathcal{L}}{\partial m^{\prime}}\right)-\frac{\partial \mathcal{L}}{\partial m}=0,
$$

reproduces the modified TOV equation (14) where we use the relation $m^{\prime}=4 \pi r^{2} \rho$.

\subsection{Anisotropic matter when $w_{1}=-1$}

The entropy density for anisotropic matter depends on the energy density $\rho(r)=4 \pi r^{2} m^{\prime}(r)$. Thus the Lagrangian for perfect fluid (35) can be generalized to the form:

$\mathcal{L}_{a b}=c\left(m^{\prime}\right)^{a} r^{b}\left(1-\frac{2 m}{r}\right)^{-\frac{1}{2}}$,

where $a, b, c$ are constants to be determined. The Euler equation,

$$
\begin{aligned}
\frac{m^{\prime 2}}{\mathcal{L}_{a b}} \times\left(\frac{d}{d r} \frac{\partial \mathcal{L}_{a b}}{\partial m^{\prime}}-\frac{\partial \mathcal{L}_{a b}}{\partial m}\right)=0 \Rightarrow a(a-1) m^{\prime \prime} \\
+\frac{a b}{r} m^{\prime}+\frac{(a-1) m^{\prime 2}-a \frac{m m^{\prime}}{r}}{r-2 m}=0,
\end{aligned}
$$

can be rewritten as a form of modified TOV equation:

$$
\begin{aligned}
p_{1}^{\prime}= & -\frac{\frac{w_{1}}{1-a} \rho}{r(r-2 m)}\left(m+\frac{1-a}{a w_{1}} 4 \pi r^{3} p_{1}\right) \\
& +\frac{\frac{b}{1-a}-2}{r} w_{1} \rho .
\end{aligned}
$$

Comparing this with the modified TOV equation (14) one obtains:

$a=\frac{1}{1+w_{1}}, b=\frac{2 w_{2}}{1+w_{1}}$.

For $w_{1} \neq-1$, the Lagrangian exactly corresponds to the one for the entropy density (33) obtained in Sect. 3, hence confirms our result.

For $w_{1}=-1$, the solution was explicitly obtained in Ref. [29]. It is sufficient that we use the results from the article:

$m(r)=M+\frac{K}{2 r^{2 w_{2}-1}}$,

$\rho(r)=-p_{1}(r)=\frac{\left(1-2 w_{2}\right) K}{8 \pi r^{2+2 w_{2}}}$,

where $M$ and $K$ are constants. Putting this information into Eq. (40) we get $a$ and $b:{ }^{4}$

$a=1-\frac{1}{2 w_{2}}, \quad b=2(a-1) w_{2}$.

Therefore, the entropy density for $w_{1}=-1$ which satisfy the maximum entropy principle is

$s=s_{0} \rho^{1-\frac{1}{2 w_{2}}} r^{-\frac{1+w_{2}}{w_{2}}}$,

where $s_{0}$ is a constant.

As we discussed in the previous section, one can determine 'the compensation factor' $\Phi_{w}(r)$ for the above entropy

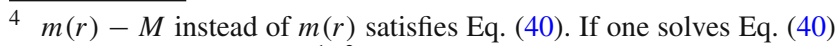
directly one gets $m(r) \propto r^{1-2 w_{2}}$, after that one can freely add a constant mass. This means $m(r)$ in Eqs. (39) and (40) is related to the physical mass up to an additional constant. 
density. When $w_{1}=-1$, to avoid the denominator to vanish, one can choose the isotropic part of the pressure as $p_{\text {iso }} \equiv p_{1}-2 p_{2}$ or $w_{\text {iso }}=w_{1}-2 w_{2}\left(=-1-2 w_{2}\right)$. The entropy density using the anisotropic factor (28) is obtained to be

$s \propto \Phi_{w}(r) \rho^{-\frac{1}{2 w_{2}}} r^{-\frac{\left(1+3 w_{2}\right)}{w_{2}}}$,

by putting $w=-1-2 w_{2}$ to Eq. (29). After requiring MEP, one obtains the factor $\Phi_{w}(r)=r^{-2 w_{2}}$ up to a multiplicative constant. By using the relation $\rho(r) \propto r^{-2\left(1+w_{2}\right)}$ in Eq. (43), one reproduces the entropy in Eq. (45).

\section{Total entropy and applications}

Total entropy of self-gravitating radiation confined to a box of radius $R$ was obtained in Ref. [8]. By using the same method, we obtain a general form of total entropy of anisotropic matter confined to the box:

$$
\begin{aligned}
S & =\left.S_{A}(r)\right|_{r=0} ^{r=R} \\
& =\left.\frac{(4 \pi)^{\frac{w_{1}}{1+w_{1}}} \alpha_{w}}{\left(1+w_{1}+2 w_{2}\right)} \frac{\frac{m}{r}+w_{1} \frac{d m}{d r}}{\left(\frac{d m}{d r}\right)^{\frac{w_{1}}{1+w_{1}}}\left(1-\frac{2 m}{r}\right)^{\frac{1}{2}}} r^{\frac{1+w_{1}+2 w_{2}}{1+w_{1}}}\right|_{r=0} ^{r=R} .
\end{aligned}
$$

One can verify that the derivative of the function on the righthand side is the Lagrangian (37) by using the relation (40). We call the indefinite integral $S_{A}(r)$ as 'entropy function'. One can see that the above total entropy reproduces that in Ref. [8] when $w_{1}=w_{2}=w_{3}=1 / 3$.

In a general case, solutions of the Einstein equation can be singular at the origin. In order for a solution to be regular at $r=0, m(r) \sim\left(4 \pi r^{3} / 3\right) \rho(0) \sim r^{3}$, and $\rho(r) \sim r^{\alpha}$ with $\alpha>0$. Otherwise the solution will be singular at the center. To analyze the singularity more explicitly, one needs to have an exact solution. The properties of solutions with $w_{1}=-1$ at $r=0$ is extensively analyzed in Ref. [29] with the exact solutions in Eq. (43).

The entropy function above can be further simplified to

$S_{A}(r)=\frac{m+4 \pi r^{3} p_{1}}{\left(1-\frac{2 m}{r}\right)^{\frac{1}{2}}}\left(\frac{s_{A}(r)}{\rho+p_{1}+2 p_{2}}\right)$

by using $m^{\prime}=4 \pi r^{2} \rho$ and the relation (4) and (33).

Recently, in Ref. [35] the author suggested conditions to avoid naked singularities for a system with an anisotropic fluid. One of the conditions is $\rho+p_{1}+2 p_{2}<0$, which indicates a violation of the strong energy condition. Putting this into the relations (47) or (48), the entropy function takes a negative value. Since entropy is defined by a possible number of physical configurations of a system, negative value of entropy seems unreasonable. If we look into the derivation of total entropy carefully, the total entropy, $\int_{a}^{b} \mathcal{L}_{A}=S_{A}(b)-S_{A}(a)$, is always non-negative since the entropy function $S_{A}(r)$ is monotonically increasing function of $r$ even if $S_{A}(r)<0$. It is because the Lagrangian density, $\mathcal{L}_{A}=S_{A}^{\prime}(r)$, is positive. Therefore, even though $\rho+p_{1}+2 p_{2}<0$, the total entropy of the system is positive.

A wormhole is a solution of general relativity that has a throat and two sides of entrance and exit [36-38]. In general relativity, the throat of a traversable wormhole needs to be made of exotic, anisotropic material which violates energy conditions (especially null energy condition [39] along with weak, strong and dominant energy conditions). If anisotropic matter (that creates a wormhole) is bound $(r<R)$ and the wormhole's throat is located at $r=B$, the entropy of the wormhole will be given by

$S=2\left[S_{A}(R)-S_{A}(B)\right]$,

provided that both sides of the wormhole have the same shape.

Morris and Thorne [30] suggested specific wormhole solutions which use exotic material minimally: (1) a zero-tidal force solutions, (2) a solution with a finite radial cutoff of the stress-energy and (3) a solution with exotic matter limited to the throat vicinity. The first and the second solutions satisfy the equation of state of type $p_{k}=w_{k} \rho$ with $1+w_{1}+2 w_{2}=0$. Although the equation of state of the third solution does not have the linear form, the matter still satisfy $\rho+p_{1}+2 p_{2}=0$. Putting these conditions into the total entropy formula (47), (48), one immediately gets indefinite value for the entropy function. From the Einstein equation for the metric (12), one gets

$$
\begin{aligned}
8 \pi\left(\rho+p_{1}+2 p_{2}\right)= & \left(1-\frac{2 m}{r}\right) \\
& \times\left(\frac{2}{r} u^{\prime}-\frac{v^{\prime} u^{\prime}}{2}+\frac{u^{\prime 2}}{2}+u^{\prime \prime}\right) .
\end{aligned}
$$

When $u^{\prime}=0$ throughout the region where exotic matter is used, $\rho+p_{1}+2 p_{2}=0$. The three examples in Ref. [30] is the case. For the same metric, the entropy function can be written as

$S_{A}(r)=\frac{4 \pi r^{2}}{\left(1-\frac{2 m}{r}\right)^{\frac{1}{2}}} \frac{s_{A}}{\left(\frac{2}{r}-\frac{v^{\prime}}{2}+\frac{u^{\prime}}{2}+\frac{u^{\prime \prime}}{u^{\prime}}\right)}$,

where we use the relation (13). We may slightly perturb the above wormhole solutions to avoid the divergence of the entropy function. If we compare two configurations of $S\left(u^{\prime}=0\right)$ and $S\left(\left|u^{\prime}\right| \neq 0\right)$, one may tell which configuration is more favourable than the other. For a smooth function $u^{\prime}(r)\left(\left|u^{\prime}\right| \ll 1\right.$ with dimension of inverse length) near the throat vicinity, the quantities $\left(u^{\prime}\right)^{2}, u^{\prime \prime}$ are much smaller than $u^{\prime}$. The denominator of the above equation reduces to 
The denominator $\propto e^{v} \frac{2 r-3 m-r m^{\prime}}{r^{2}}+\frac{u^{\prime}}{2}$.

The sign of the first term may switch from positive to negative and vice versa. For example, in the third wormhole in Ref. [30] the mass was given by $m(r)=b[1-(r-b) / a]^{2}$, where $u^{\prime}=0$ in the region $b \leq r \leq b+a$. Provided that perturbations around $u^{\prime}=0$ is small and the solution of $m(r)$ has the same quadratic form, occasional sign changes appear for various values of $a$ and $b$. The entropy function becomes extremely large when the denominator approaches zero. When there is a sign change, the indefinite value makes comparison difficult.

As we discussed in Sect. 3, the freedom of choice in isotropic pressure part may help us to prevent from this pathology. When we set the isotropic pressure part as $\mathcal{P} \equiv$ $p_{1}+2 p_{2}$, the process of getting the anisotropic factor $\phi$ suffers the same pathology when $\rho+p_{1}+2 p_{2}=0$ or $w_{1}+2 w_{2}=-1$ as in the case when $w_{1}=-1$. We expect that with proper 'compensation factor' $\Phi_{w}$ one can obtain finite form of entropy density by using a similar process in Sect. 4.3.

The thermodynamic stability of the configuration $u^{\prime}=0$ can be estimated by the difference of the entropy functions, $\Delta S=\left[S_{A}\left(r, u^{\prime}=0\right)-S_{A}\left(r, u^{\prime} \neq 0\right)\right]_{a}^{b}$. To this end, further analysis with wider range of exact solutions including $u^{\prime} \neq 0$ will be necessary.

Incidentally, we get another form of the entropy function

$S_{A}(r)=-4 \pi \frac{\left(m+4 \pi r^{3} p_{1}\right)}{\sqrt{1-\frac{2 m}{r}} R_{0}^{0}} s_{A}$.

using $R_{0}^{0}=-4 \pi\left(\rho+p_{1}+2 p_{2}\right)$.

\section{Summary and discussions}

In this article we considered a system of self-gravitating, static and spherically symmetric matter of which pressure satisfies the linear equation of state $p_{1}=w_{1} \rho$ and $p_{2}=$ $p_{3}=w_{2} \rho$. We obtained a form of entropy density for the anisotropic matter. To obtain the exact form of entropy density, we introduced a scalar function $\phi(x)$, the anisotropic factor that contains the information of anisotropic deviation from an isotropic perfect fluid. The choice of an isotropic part of the pressure was shown to have an arbitrariness. A compensation vector was obtained to gauge this arbitrariness. This gauge freedom could be beneficial when we meet a problem. In the process, the maximum entropy principle (MEP) played a key role in obtaining the exact form of the entropy. We showed that the requirement of local maximum of total entropy gives the exact Einstein equation of the system composed of the anisotropic matter. This observation supports the correspondence between thermodynamics and gravity. By the guidance of this correspondence, the entropy density for $p_{1}=-\rho$ or $w_{1}=-1$ was obtained separately.

The entropy density in this article has a multiplicative factor that describes the anisotropic effect. The quantity we obtained is a function of radius $r$ and $w_{2}-w_{1}$. This comes from the use of the spherical coordinate system and the simple form of anisotropy between the radial and the angular directions. In a more general situation, the entropy density is expected to have various forms as a function of energy density and pressure.

With the explicit form of the total entropy function (47), as an application, we tried to analyze a wormhole solution by estimating the entropy in the vicinity of a throat. Actually, most of the issues about wormholes come from the fact that matter composing wormholes are not ordinary but exotic. There are various ways to overcome or to go around this issue. For a modified gravity theory, one may find wormholes without exotic matter. There were many articles in which a throat of traversable wormholes is composed of ordinary matter in various modified gravity theories, e.g., Einstein-GaussBonet theory [40] or Lovelock theory [41], $f(R)$ gravity [42], etc. [43,44]. Similar analysis on the entropy for the wormholes in those theories is worth trying. Especially, the method in this article can be applicable to an anisotropic matter in the context of $f(R)$ gravity, because the MEP was proven for self-gravitating fluid in $f(R)$ gravity [45]. On the other hand, if we stick to the Einstein gravity, our result shows that building materials or conditions should be chosen carefully.

There are many studies on astro-physical objects such as relativistic stars, neutron stars, etc. in which anisotropic pressure takes important roles $[21,24,46]$. We expect that the entropy obtained in this article can be used to estimate the state of a relativistic star by comparing the values of entropy for different configurations of the star. The selfgravitating solutions for the anisotropic matter were classified in Ref. [47]. For the case of matter with non-linear equation of states, the analysis will be quite difficult and deeper studies on the entropy formula will be required.

Acknowledgements This work was supported by the National Research Foundation of Korea Grants funded by the Korea government NRF-2017R1A2B4008513. Y. Lee is grateful to Dr. Ma, Chung-Hyeun for his kind treatment in the hospital.

Data Availability Statement This manuscript has no associated data or the data will not be deposited. [Authors' comment: The article is purely theoretical.]

Open Access This article is distributed under the terms of the Creative Commons Attribution 4.0 International License (http://creativecomm ons.org/licenses/by/4.0/), which permits unrestricted use, distribution, and reproduction in any medium, provided you give appropriate credit to the original author(s) and the source, provide a link to the Creative Commons license, and indicate if changes were made.

Funded by SCOAP ${ }^{3}$. 


\section{References}

1. S.W. Hawking, Commun. Math. Phys. 43, 199 (1975) Erratum: [Commun. Math. Phys. 46 (1976) 206]. https://doi.org/10.1007/ BF02345020, https://doi.org/10.1007/BF01608497

2. J.D. Bekenstein, Phys. Rev. D 9, 3292 (1974). https://doi.org/10. 1103/PhysRevD.9.3292

3. T. Jacobson, Phys. Rev. Lett. 75, 1260 (1995). https://doi.org/10. 1103/PhysRevLett.75.1260. arXiv:gr-qc/9504004

4. T. Padmanabhan, Rep. Prog. Phys. 73, 046901 (2010). https://doi. org/10.1088/0034-4885/73/4/046901. arXiv:0911.5004 [gr-qc]

5. E.P. Verlinde, JHEP 1104, 029 (2011). https://doi.org/10.1007/ JHEP04(2011)029. arXiv:1001.0785 [hep-th]

6. S. Carlip, Int. J. Mod. Phys. D 23, 1430023 (2014). https://doi.org/ 10.1142/S0218271814300237. arXiv:1410.1486 [gr-qc]

7. W.J. Cocke, Ann. Inst. Henri Poincaré 2, 283 (1965)

8. R.D. Sorkin, R.M. Wald, Z.Z. Jiu, Gen. Rel. Grav. 13, 1127 (1981)

9. R.C. Tolman, Phys. Rev. 55, 364 (1939). https://doi.org/10.1103/ PhysRev.55.364

10. J.R. Oppenheimer, G.M. Volkoff, Phys. Rev. 55, 374 (1939). https:// doi.org/10.1103/PhysRev.55.374

11. S. Gao, Phys. Rev. D 84, 104023 (2011) (Addendum: [Phys. Rev. D 85 (2012) 027503]). https://doi.org/10.1103/PhysRevD. 84.104023, https://doi.org/10.1103/PhysRevD.85.027503. arXiv:1109.2804 [gr-qc]

12. Z. Roupas, Class. Quant. Grav. 30(11), 115018 (2013) Erratum: [Class. Quant. Grav. 32 (2015) 119501]. https://doi.org/10.1088/ 0264-9381/32/11/119501, https://doi.org/10.1088/0264-9381/ 30/11/115018, arXiv:1411.0325 [gr-qc], arXiv:1301.3686 [gr-qc]

13. L.M. Cao, J. Xu, Z. Zeng, Phys. Rev. D 87(6), 064005 (2013). https://doi.org/10.1103/PhysRevD.87.064005. arXiv:1301.0895 [gr-qc]

14. S. Weinberg, Gravitation and Cosmology: Principles and Applications of the General Theory of Relativity, Chapter 10

15. W.H. Zurek, D.N. Page, Phys. Rev. D 29, 628 (1984). https://doi. org/10.1103/PhysRevD.29.628. arXiv:1511.07051 [gr-qc]

16. P.H. Chavanis, Astron. Astrophys. 483, 673 (2008). https://doi.org/ 10.1051/0004-6361:20078287. arXiv:0707.2292 [astro-ph]

17. H. Stephani, D. Kramer, M.A.H. MacCallum, C. Hoenselaers, E. Herlt. https://doi.org/10.1017/CBO9780511535185

18. M.S.R. Delgaty, K. Lake, Comput. Phys. Commun. 115, 395 (1998). https://doi.org/10.1016/S0010-4655(98)00130-1. arXiv:gr-qc/9809013

19. I. Semiz, Rev. Math. Phys. 23, 865 (2011). https://doi.org/10.1142/ S0129055X1100445X. arXiv:0810.0634 [gr-qc]

20. M. Ruderman, Ann. Rev. Astron. Astrophys. 10, 427 (1972). https://doi.org/10.1146/annurev.aa.10.090172.002235

21. L. Herrera, N.O. Santos, Phys. Rep. 286, 53 (1997). https://doi. org/10.1016/S0370-1573(96)00042-7

22. R.L. Bowers, E.P.T. Liang, Astrophys. J. 188, 657 (1974). https:// doi.org/10.1086/152760

23. J.J. Matese, P.G. Whitman, Phys. Rev. D 22, 1270 (1980). https:// doi.org/10.1103/PhysRevD.22.1270

24. M.K. Mak, T. Harko, Proc. R. Soc. Lond. A 459, 393 (2003). https:// doi.org/10.1098/rspa.2002.1014. arXiv:gr-qc/0110103
25. S. Thirukkanesh, S.D. Maharaj, Class. Quant. Grav. 25, 235001 (2008). $\quad$ https://doi.org/10.1088/0264-9381/25/23/235001. arXiv:0810.3809 [gr-qc]

26. B.V. Ivanov, Phys. Rev. D 65, 104011 (2002). https://doi.org/10. 1103/PhysRevD.65.104011. arXiv:gr-qc/0201090

27. V. Varela, F. Rahaman, S. Ray, K. Chakraborty, M. Kalam, Phys. Rev. D 82, 044052 (2010). https://doi.org/10.1103/PhysRevD.82. 044052. arXiv: 1004.2165 [gr-qc]

28. J.D. Bekenstein, Phys. Rev. D 4, 2185 (1971). https://doi.org/10 1103/PhysRevD.4.2185

29. I. Cho, H.C. Kim. arXiv:1703.01103 [gr-qc]

30. M.S. Morris, K.S. Thorne, Am. J. Phys. 56, 395 (1988). https:// doi.org/10.1119/1.15620

31. M. Cataldo, L. Liempi, P. Rodríguez, Phys. Lett. B 757, 130 (2016). https://doi.org/10.1016/j.physletb.2016.03.057. arXiv: 1604.04578 [gr-qc]

32. S.A. Hayward, Relativistic Thermodynamics, gr-qc/9803007

33. H. Stephani, J. Steward (Cambridge, Cambridge University Press, 1982) (Transl. from German By M. Pollock and J. Stewart)

34. K. Schatz, H.H. von Borzeszkowski, T. Chrobok, J. Grav. 2016, 4597905 (2016). https://doi.org/10.1155/2016/4597905

35. H.C. Kim, Phys. Rev. D 96(6), 064053 (2017). https://doi.org/10. 1103/PhysRevD.96.064053. arXiv:1708.02373 [gr-qc]

36. H. Weyl, Feld und Materie. Annalen der Physik. 65(14), 541-563 (1921)

37. C.W. Misner, J.A. Wheeler, Ann. Phys. 2, 525 (1957). https://doi. org/10.1016/0003-4916(57)90049-0

38. S.W. Kim, H. Lee, Phys. Rev. D 63, 064014 (2001). https://doi. org/10.1103/PhysRevD.63.064014. arXiv:gr-qc/0102077

39. A. Raychaudhuri, Phys. Rev. 98, 1123 (1955). https://doi.org/10. 1103/PhysRev.98.1123

40. M.R. Mehdizadeh, M. Kord Zangeneh, F.S.N. Lobo, Phys. Rev. D 91(8), 084004 (2015). https://doi.org/10.1103/PhysRevD.91. 084004. arXiv:1501.04773 [gr-qc]

41. M. Kord Zangeneh, F.S.N. Lobo, M.H. Dehghani, Phys. Rev. D 92(12), 124049 (2015). https://doi.org/10.1103/PhysRevD.92. 124049. arXiv: 1510.07089 [gr-qc]

42. S.H. Mazharimousavi, M. Halilsoy, Mod. Phys. Lett. A 31(34), 1650192 (2016). https://doi.org/10.1142/S0217732316501923

43. K.K. Nandi, A. Islam, J. Evans, Phys. Rev. D 55, 2497 (1997). https://doi.org/10.1103/PhysRevD.55.2497. arXiv:0906.0436 [grqc]

44. E.F. Eiroa, M.G. Richarte, C. Simeone, Phys. Lett. A 373, 1 (2008) Erratum: [Phys. Lett. 373 (2009) 2399] https://doi.org/10.1016/j. physleta.2008.10.065, https://doi.org/10.1016/j.physleta.2009.04. 065. arXiv:0809.1623 [gr-qc]

45. X. Fang, M. Guo, J. Jing, JHEP 1608, 163 (2016). https://doi.org/ 10.1007/JHEP08(2016)163. arXiv:1512.05454 [gr-qc]

46. A.A. Isayev, Phys. Rev. D 96(8), 083007 (2017). https://doi.org/ 10.1103/PhysRevD.96.083007. arXiv: 1801.03745 [gr-qc]

47. H.C. Kim, Phys. Rev. D 95(4), 044021 (2017). https://doi.org/10. 1103/PhysRevD.95.044021. arXiv:1601.02720 [gr-qc] 